\section{The retinal threshold gradient in the presence of a high-luminance target and in total darkness*}

\author{
RICHARD F. HAINES \\ Ames Research Center, NASA Moffett Field, California 94035
}

The retinal threshold was measured along the horizontal meridian for the dark-adapted eye and for the same retinal region light-adapted by stray light from a small foveally fixated high-luminance target. This was done to quantify the irradiation phenomenon in the foveal-parafoveal boundary region. Two target luminances were investigated $(5,531$ and 13,000 fL). Seven Ss made threshold settings using a variable-intensity test spot whose retinal image was located at various angular separations from the foveal center as far as $10 \mathrm{deg}$ in the temporal field. Threshold slopes were steeper for test spots imaged from $15 \mathrm{~min}$ to $1 \mathrm{deg}$ of arc from the foveal center than for spots imaged from $1 \mathrm{deg} 15 \mathrm{~min}$ to $10 \mathrm{deg}$ of arc from the foveal center for the illuminated target conditions. The angular distance between the fovea and the intersection of the steep component with the shallow component of each threshold curve tended to shift toward the target's edge with an increase in target luminance. These data are interpreted as an indication that a dynamic neural mechanism is involved in producing the irradiation phenomenon.

When two adjacent areas of the visual field are at different luminances and are separated by a discontinuous (step) luminance distribution, the apparent edge between them shifts toward the dimmer area (Haines, 1969a; Helmholtz, 1962; Luckiesh \& Moss, 1931; Plateau, 1838). Further quantification of this "irradiation phenomenon" is one objective of the present investigation. In this situation Mach bands sometimes become apparent (Matthews, 1966; Ratliff, 1965), the brighter area appears larger than it actually is (Haines, 1965, 1969a; Haines \& Allen, 1968; Haines \& Bartley, 1966), and the brighter area (hereafter called the target) can cause the dimmer area (hereafter called the background) to appear darker than it is (simultaneous contrast).

These perceptual phenomena are produced by optical, neural, photochemical, and psychological mechanisms. The present paper deals with the first two of these mechanisms. Research on entoptic scattering has shown that the bundle of photic energy from a relatively high-contrast ${ }^{1}$ target scatters a tapered veiling illumination over the entire retina (Bartley \& Fry, 1934; Boynton, Enoch, \& Bush, 1954; DeMott \& Boynton, 1958; Fry \& Alpern, 1953; LeGrand, 1937). Another source of entoptic illumination is the light that is reflected off the retina within the target's image

*The author wishes to thank Mr. Lewis B. Mayhew, Jr. for his assistance in collecting portions of this data and to Mary M. Connors and Thomas Cornsweet for their valuable comments. fovea. Vos (1963) points out, "... the graphs cannot be explained in terms of entoptic stray light only. It is clear on the other hand, from the strictly linear relationship at large (glare) angles, that stray light is an important factor. It seems improbable, that the scatter mechanism changes, on physical grounds and because the inflexions lie at one level of $B_{e q}$, rather than $\mathrm{E}$. A change in receptor mechanism seems to be a more attractive assumption ... the Purkinje shift ... the transition from rod to cone vision [p. 14]."

In the psychophysical studies on the effects of veiling luminance which Vos (1963) reviews, the glare source is systematically imaged across the retina while the test spot remains imaged on the fovea. It would be impossible for studies that use this measurement technique to obtain a threshold inflexion as a function of test spot location. It is important to note, however, that small threshold inflexions have been reported in other psychophysical studies where the test spot was systematically imaged across the retina in the foveal-parafoveal boundary region, e.g., absolute and differential visual threshold (deGroot, Dodge, \& Smith, 1952), chromatic threshold (Wentworth, 1930), acuity (Katzenellenbogen, 1907; Mandelbaum \& Sloan, 1947). However, Weymouth, Hines, Acres, Raaf, and Wheeler (1928) investigated acuity in this retinal region and did not find such an inflexion.

The evidence that shows a threshold inflexion in the region of the foveal-parafoveal boundary leads to the second objective of this investigation, namely, to obtain more complete threshold data for this region of the retina. Interestingly, no one has studied the light threshold response of this region in detail. Kristofferson (1955) quantified foveal sensitivity to a small flashing test spot imaged at each of eight angular locations from $0 \mathrm{deg}$ to $40 \mathrm{~min}$ of arc from the foveal center along each of three meridians. According to Osterberg's (1935) and Polyak's (1941) histological measurements, however, the horizontal dimension of the human rod-free fovea is just under $2 \mathrm{deg}$, while the vertical dimension is somewhat less (1 deg $45 \mathrm{~min}$ of arc). If these dimensions are accepted, Kristofferson's (1955) largest test spot separation from the foveal center was still within the pure cone region (except for possible occasional shifts in test spot location due to involuntary ocular motion). Other investigators have quantified retinal sensitivity in 1-deg-arc steps apart or larger, starting about $1 \mathrm{deg}$ of arc from the center of the fovea (Crozier \& Holway, 1939; deGroot et al, 1952; Pirenne \& Marriott, 1954; Riopelle \& 


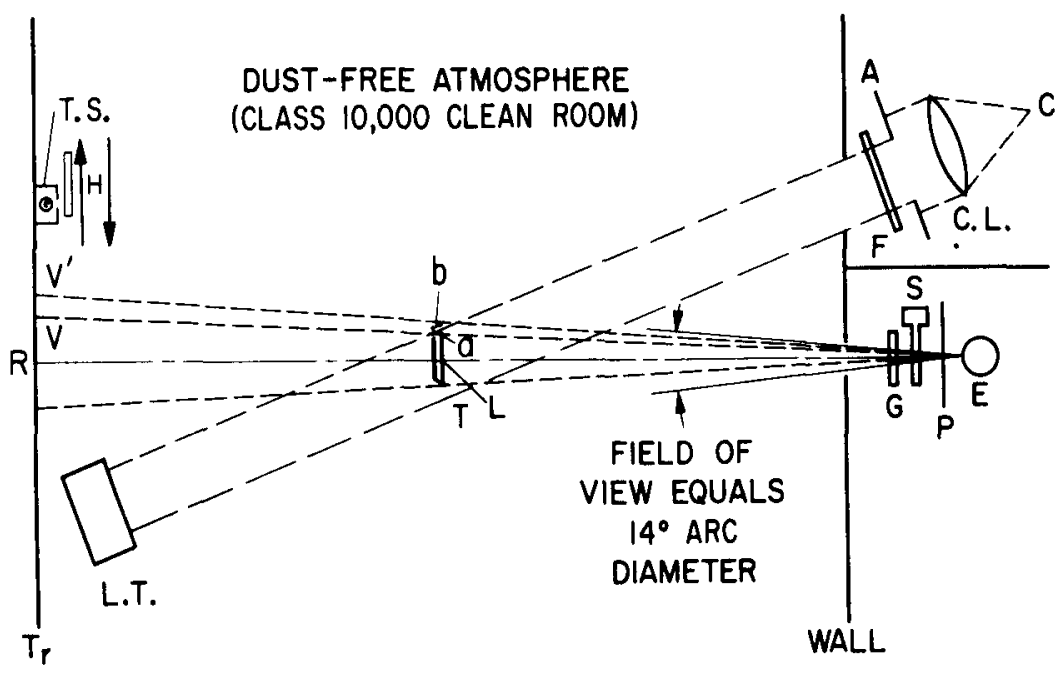

Fig. 1. Schematic diagram of testing facility and equipment.

Bevan, 1953; Sperling \& Farnsworth, 1950). Because these investigators studied retinal areas so far apart from one another, it is unilikely that their data could exhibit a differential threshold effect in the foveal-parafoveal boundary region.

The methodology used in the present investigation provides a means of quantifying both the absolute threshold gradient and the threshold gradient resulting from the bright target's veiling retinal illumination. Any differences found between these gradients should help clarify the role played by optical and neural mechanisms that underlie the irradiation in the foveal and parafoveal region.

\section{METHOD}

\section{Apparatus}

The apparatus and testing facility is described elsewhere (Haines \& Allen, 1968; Haines \& Burgard, 1967) and is only summarized here. The basic equipment is shown in Fig. 1. The S's right eye (E) foveally fixates the center of a 48-min-arc diameter circular $\mathrm{M}_{\mathrm{g}} \mathrm{O}$-coated flat target (T) against an unilluminated background. The beam from a carbon arc $\left(5,800^{\circ} \mathrm{K}\right)$ solar simulator (C) illuminates the target after first passing through a collimating lens (CL), an aperture (A), and an inconel neutral density filter (F). The target luminances investigated were 5,531 and $13,000 \mathrm{fL}$. A light trap (LT) captures light that passes the target. Just in front of the eye is a field stop (P) which produces a 14-deg-arc diameter field of view, a shutter (S), and an inconel neutral density filter (G). A track (Tr) lay directly behind the center of the target (L); the center of the track (R) lay on an imaginary line (E-L-R). A servodriven carriage travels on this track. On the carriage is a $\left(1,900^{\circ} \mathrm{K}\right)$ test spot (TS) that subtends $10 \mathrm{sec}$ of arc and has a maximum intensity of $141 \mathrm{mlm}$. A circular inconel neutral density filter $(\mathrm{H})$, located just in front of the test spot, is used to vary its intensity. Walls separate the S's cubicle, solar simulator, and the class 10,000 clean room.

\section{Procedure}

The test spot, which the $S$ uses to determine his threshold, is positioned by $\mathrm{E}$ into each of the angular separations, $\theta$, of interest from the target's right-hand edge. For the data given in Fig. 2, the 9 test spot positions were presented randomly. Each $S$ made 15 brighter and 15 dimmer test spot settings, alternately, at each $\theta$ position. For the data given in Fig. 3 , the test spot positions were presented sequentially (i.e., the test spot traveled from right to left during one session and from left to right during the next, etc.) a total of 10 times each direction. Thus, each data point is based upon 20 test spot settings. The horizontal meridian on the temporal side of the fovea was investigated.

The viewing shutter opened for $15 \mathrm{sec}$ every trial to expose the target and test spot. A trial lasted $29 \mathrm{sec}$. The E set the test spot's intensity at random levels prior

To help S maintain as stable a fixation as possible during the unilluminated target portion of the study, $S$ fixated a dim (about $1 \log _{10}$ unit above his photopic threshold) red, 1-min-arc-diam spot of light, projected onto the target's center. The $S$ was instructed to maintain fixation on the target's center for all of the results presented here except for the middle curve of Fig. 3. The $S$ was told to fixate the test spot for this condition. Apparatus limitations prevented collection of data beyond $\theta=6 \mathrm{deg}$ for this condition.

Since previous studies (Haines, 1969b; to each trial.
Spring \& Stiles, 1948) have shown that a correction of less than $0.02 \log _{10}$ unit is required to take into account the reduction in apparent pupil area when the test spot is located at angles up to $10 \mathrm{deg}$ of arc from the line of sight, this factor need not be taken into account.

Point a (see Fig. 1) represents the perceived edge of the target at relatively low luminances, while Point $b$ represents the location of the apparent edge under high luminances. The magnitude of this irradiation shift is determined by solving for angle V-E- $V^{\prime}$ when distances V-E and $\mathrm{V}-\mathrm{V}^{\prime}$ are known.

\section{Subjects}

Seven males took part. Their ages ranged from 19 to 31 years (mean $=23$ years). All had 20:20 uncorrected distance acuity except RH, who had 20:20 corrected distance acuity. All had normal color perception (Ishihara and A.O Pseudo-Isochromatic Plates), full visual fields, and normal ocular motility. All were given sufficient training in the task to preclude learning effects.

\section{RESULTS AND DISCUSSION}

The mean threshold gradient for each $\mathrm{O}$ and experimental condition is given in Figs. 2 and 3 . The shaded vertical bar represents the relative position and angular width of the target. The abscissa represents the angular separation $\theta$ between the test spot and right-hand edge of the target. All of the curves drawn through the data points and the slopes given in parentheses near each component, except for the absolute threshold for Fig. 3, refer to a linear least-squares fit to: (a) the $0 \mathrm{deg}<\theta<$ $1 \mathrm{deg}$, and (b) the $1 \mathrm{deg} 15 \mathrm{~min}<\theta<$ $10 \mathrm{deg}$ components, respectively. The relatively large scatter in some of the data points for MS and PE in Fig. 2 tend to reduce the meaningfulness of a linear fit; in these cases a dashed line has been drawn through these points. The curves and slopes given for the absolute threshold in Fig. 3 refer to a linear least-squares fit to: (a) the $0 \mathrm{deg}<\theta<3 \mathrm{deg}$, and (b) the $3 \mathrm{deg} 15 \mathrm{~min}<\theta<10 \mathrm{deg}$ components, respectively. Table 1 presents the mean slope of the (a) and (b) components for Fig. 2, averaged across Ss.

Table 1

Mean Slope of the (a) and (b) Components Averaged Across Observers

\begin{tabular}{|c|c|c|c|c|}
\hline & \multicolumn{3}{|c|}{ Target Luminance (fL) } \\
\hline & & Zero & 5,531 & 13,000 \\
\hline$\stackrel{\vec{E}}{0}$ & (a) & -4.14 & -3.25 & -12.03 \\
\hline ह & (b) & -1.63 & +0.11 & -0.21 \\
\hline
\end{tabular}




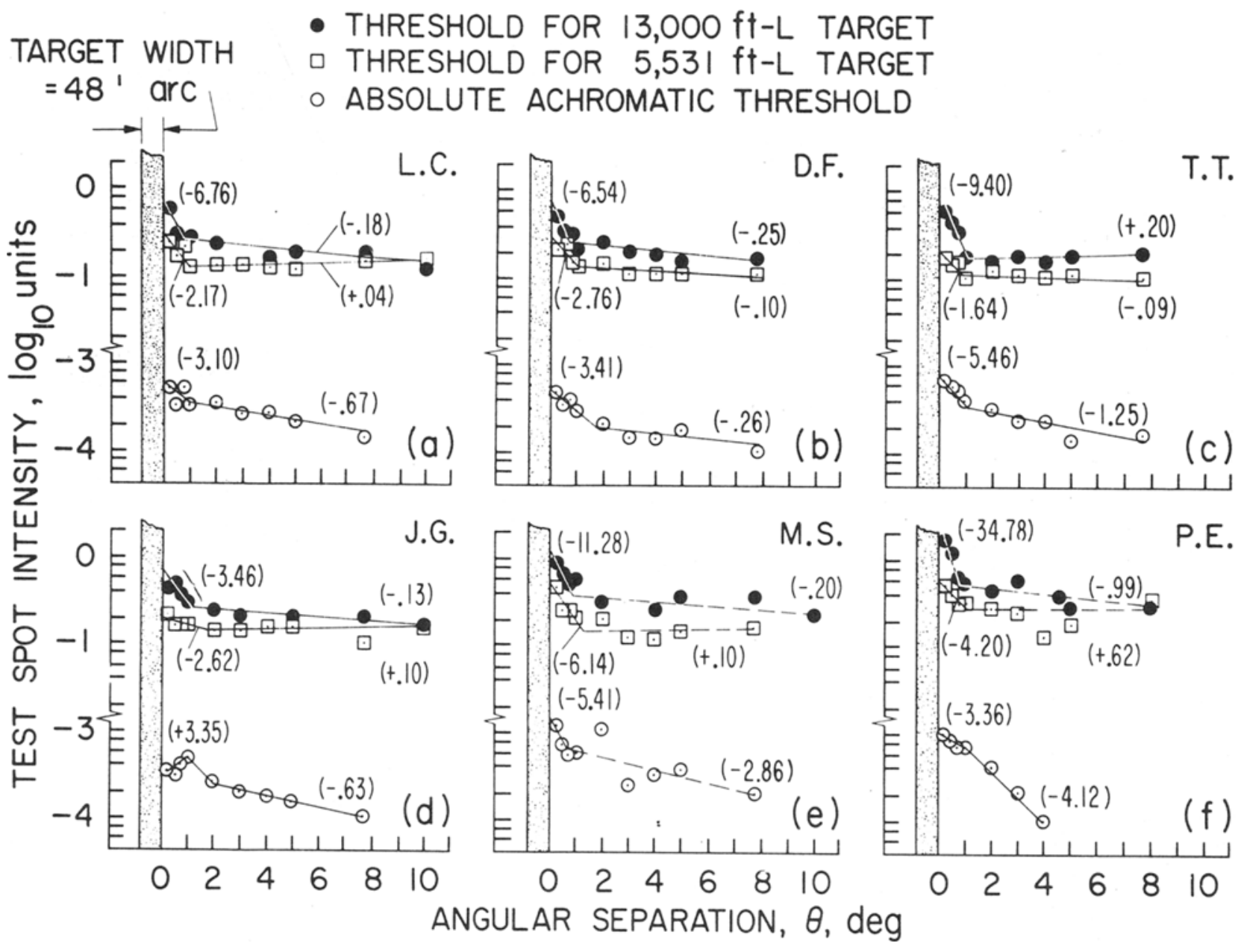

Fig. 2. Mean threshold gradient for each $S$ and experimental condition; test spot position varied randomly.

The following generalizations can be made from the data of Fig. 2: (1) For the illuminated target data, the slope of the $15 \mathrm{~min}<\theta<1 \mathrm{deg}$ component was steeper than was the slope of the 1 deg $15 \min <\theta<10$ deg component; (2) the slope of the $15 \mathrm{~min}<\theta<1 \mathrm{deg}$ component for the $13,000-\mathrm{fL}$ target luminance condition was steeper than the same component of the unilluminated target condition; (3) the slope of the $1 \mathrm{deg} 15 \mathrm{~min}<\theta<10 \mathrm{deg}$ component for the unilluminated target condition was steeper than the same component of the 13,000-fL target luminance condition; (4) the data for the two illuminated target conditions show a vertical separation of about $0.15 \log _{10}$ unit; and (5) some of the data shown in Fig. 2 (notably those of LC, DF, JG, and MS) exhibit a small inflexion at between $30 \mathrm{~min}<\theta<1$ deg $45 \mathrm{~min}$.

The threshold gradients produced by the foveally fixated, illuminated target may be described as being composed of an (a) and a (b) component. The steeper (a) component represents the retinal response from test spots imaged near the edge of the target's retinal image. It appears to involve only foveal receptors (and related nervous system) and extends to the estimated location of the rod line. This component may be the result of a neurally mediated edge-forming mechanism discussed elsewhere (Haines, 1969a; Matthews, 1966). The relatively shallow (b) component represents the retinal response from test spots imaged on the para- and perifoveal retina. ${ }^{3}$ This is the retinal region that receives a relatively constant amount of scattered illumination from the bright target. It is suggested that this component may be the result of a neurally mediated background-forming mechanism, which is discussed below. Together, these two components result in a sharpened perceived edge separating the target from the background. These data confirm a suggestion made by Matthews (1966) that the visual nervous system attempts to compensate for the tapered gradient of illumination produced by entoptic scatter by making the perceived gradient of light conform more closely to the original step distribution of retinal illumination. Table 2 gives the angular separation distance from the fovea to the point at which the (a) and (b) components intersect for each target luminance and $S$ shown in Fig. 2. It is apparent that, for all Ss except TT, for the two illuminated target conditions, this distance decreases with increases in target luminance

Regarding the vertical separation between the two uppermost curves in each graph of Fig. 2, it can be seen that once the retina has adapted to a relatively high luminance target $(5,531 \mathrm{fL})$, adding further luminance (adding 7,469 fL to equal $13,000 \mathrm{fL}$ ) merely shifts the entire threshold up by a relatively constant amount of about $0.15 \log _{10}$ unit. For the (b) component at least, it is as if some gain mechanism had changed sensitivity by a constant factor regardless of the test spot's retinal position. An important question 


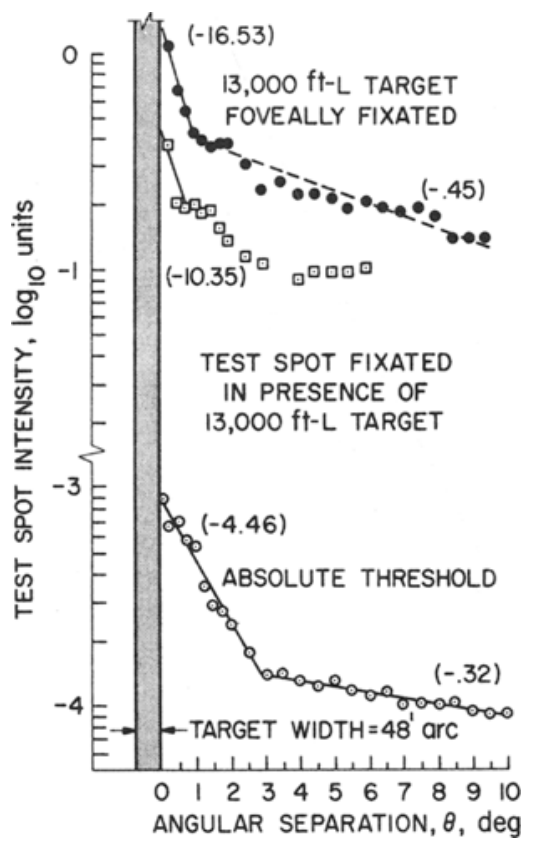

Fig. 3. Mean threshold gradient for S RH; test spot position varied sequentially across the field of view.

raised by these findings is, by what means does the retina alter its threshold sensitivity for the (a) component?

A comparison of the two uppermost curves of Fig. 3 illustrates the change in foveal threshold that is produced by moving a high luminance target across the field of view (middle curve) and the threshold at various peripheral retinal locations, produced by foveally fixating the same high luminance target (top curve). Three characteristics distinguish these two curves: (1) Each is displaced vertically from the other, (2) the (a) component slopes differ from each other by a large amount, while the (b) component slopes are almost the same, and (3) a small threshold inflexion is found at $45 \mathrm{~min}<\theta$ $<1 \mathrm{deg} 15 \mathrm{~min}$ in the middle curve and at $1 \mathrm{deg}<\theta<1 \mathrm{deg} 45 \mathrm{~min}$ in the uppermost curve. The first two characteristics can be accounted for by differences in sensitivity of the fovea and parafovea. Let us consider the third characteristic in greater detail. Figure 4 (top) is a schematic illustration of the idealized energy distribution at the retina corresponding to the uppermost curve in Fig. 3; likewise, Fig. 4 (bottom) corresponds to the middle curve of Fig. 3. The beam from the target (shaded and labeled "target width") and the idealized entoptic scatter gradient (stippled) it produces are drawn the same in both parts of this figure. $\mathrm{L}^{\prime}$ and $\mathrm{TS}^{\prime}$ refer to the center of the target's image and test spot image, respectively. The thin broken line from $\mathrm{TS}^{\prime}$
Table 2

Angular Separation Distance from the Foveal Center to the Point at Which the (a) and (b) Components Intersect

\begin{tabular}{cccccccc}
\hline & \multicolumn{7}{c}{ Target } \\
\cline { 2 - 7 } Luminance & LC & DF & TT & JG & MS & PE & Mean \\
\hline 13,000 & $45 \mathrm{~min}$ & $45 \mathrm{~min}$ & $60 \mathrm{~min}$ & $65 \mathrm{~min}$ & $60 \mathrm{~min}$ & $50 \mathrm{~min}$ & $54.2 \mathrm{~min}$ \\
5,531 & $55 \mathrm{~min}$ & $65 \mathrm{~min}$ & $55 \mathrm{~min}$ & $105 \mathrm{~min}$ & $80 \mathrm{~min}$ & $60 \mathrm{~min}$ & $70.2 \mathrm{~min}$ \\
Zero & $60 \mathrm{~min}$ & $110 \mathrm{~min}$ & $60 \mathrm{~min}$ & $120 \mathrm{~min}$ & $40 \mathrm{~min}$ & $65 \mathrm{~min}$ & $75.8 \mathrm{~min}$ \\
\hline
\end{tabular}
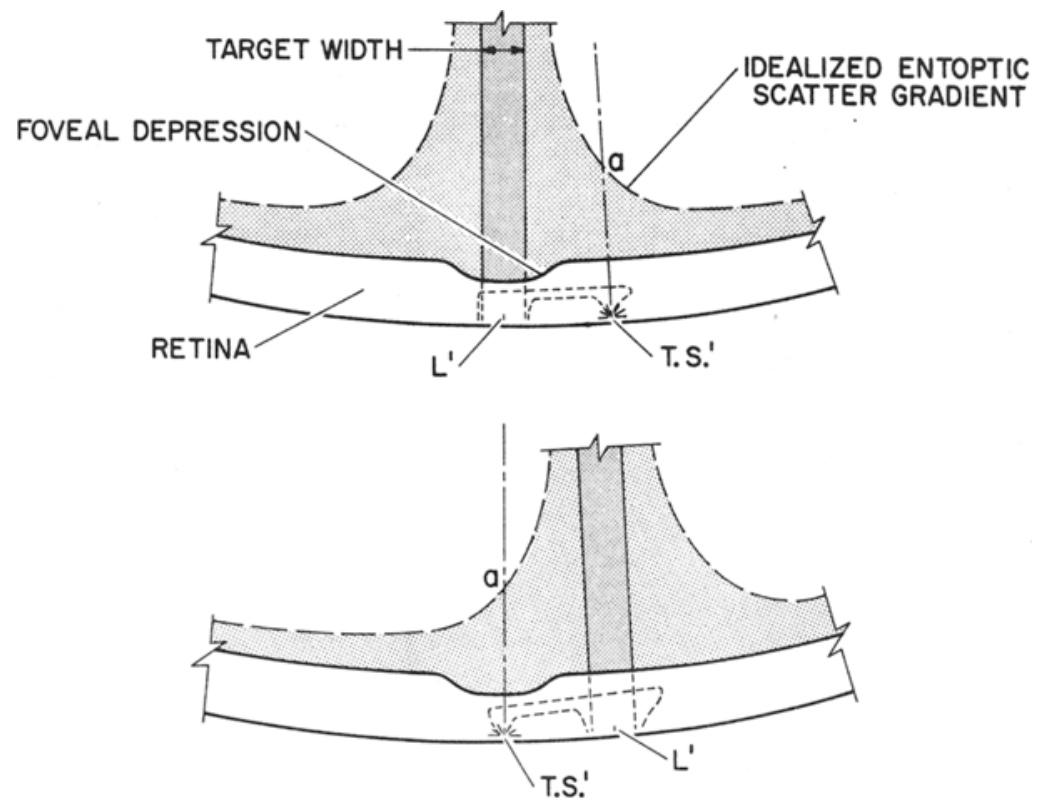

Fig. 4. Schematic illustration of the idealized energy distribution at the retina. Top corresponds to the middle curve in Fig. 3.

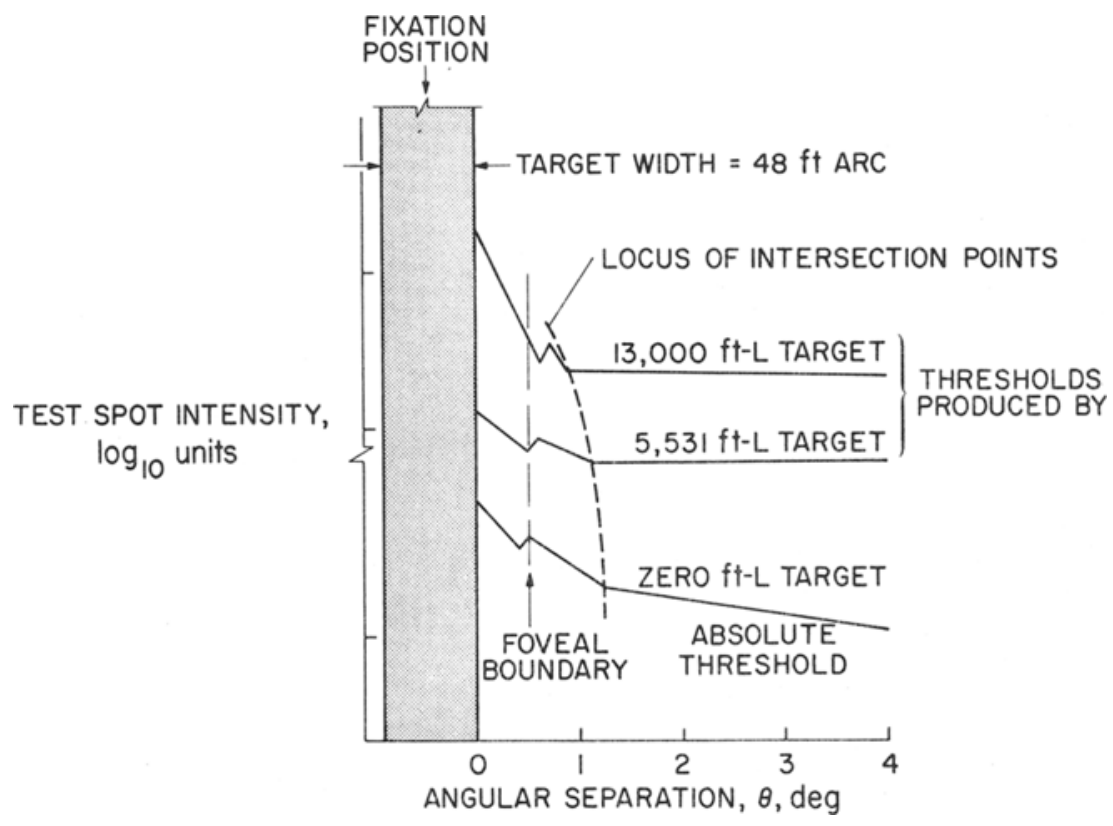

Fig. 5. Schematic diagram of how the (a) and (b) components vary with target luminance. 
and passing through "a" represents the ray from the test spot. The distance from " $a$ "

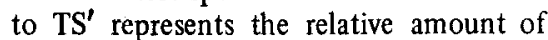
scatter falling at Point $\mathrm{TS}^{\prime}$. The dashed lines connecting $\mathrm{TS}^{\prime}$ and $\mathrm{L}^{\prime}$ with in the retina are meant to represent possible neural interactive processes.

If the unequal distance that was found between the threshold inflexion and the target's edge for the two uppermost curves in Fig. 3 is due to the amount of scattered illumination falling at Point TS', then there should be evidence to show that the distribution of scattered illumination is asymmetric about the target's beam. There is no empirical evidence that the author knows of that indicates this. In fact, there is little reason to believe that the entoptic scatter gradient is anything but symmetrical about the center of the target's beam. Thus, it is likely that this inflexion shift has a nonoptical origin.

Further evidence for the existence of a dynamic neural interactive component in the irradiation phenomenon is presented in Fig. 5. It is a schematic illustration that summarizes the results of the present investigation. The dashed curve labeled "locus of intersection points" is based upon the mean intersection distances given in Table 2 for each target luminance averaged across Ss. The systematic shift toward the target of these intersection points with an increase in target luminance suggests that a nonoptical mechanism is involved. If this effect were purely the result of an optical (scatter) phenomenon, the intersection points would become increasingly distant from the target's edge with an increase in target luminance. ${ }^{4}$ That this dynamic mechanism might be neural is not difficult to accept in light of work by Barlow, Fitzhugh, and Kuffler (1957) on the cat and by Glezer (1965) on humans, showing that receptive field dimensions decrease as adaptation level increases. Also, McCollough (1955) has found a systematic decrease in the width of the bright Mach band with an increase in target luminance.

The close correspondence that exists between the location of the threshold inflexion observed in some of the present data and in the studies cited previously suggests that a common mechanism may exist in this retinal region. Further research on the visual threshold gradient and other psychophysical and psychophysiological responses should be conducted over a range of adaptation levels and test spot image positions. Such data would help specify further the spatial and intensity relations that exist at the foveal-parafoveal boundary.

\section{REFERENCES}

BARLOW, H. B., FITZHUGH, R., \& KUFFLER, $S$. W. Change of organization in the receptive fields of the cat's retina during dark-adaptation. Journal of Physiology, 1957, 137, 338-354.

BARTLEY, S. H., \& FRY, G. A. An indirect method for measuring stray light within the human eye. Journal of the Optical Society of America, 1934, 24, 342-347.

BOUMAN, M. A. Glare: "Actio in distans" via the retina or pure contrast phenomenon? Research Unit for Perception of the National Defense Research Council, The Netherlands, Report No. WW1953-14, 1953.

BOYNTON, R. M., ENOCH, J. M., \& BUSH, W. $R$. Physical measures of stray light in excised eyes. Journal of the Optical Society of America, 1954, 44, 879-886.

BRINDLEY, G. S., \& WILLMER, E. N. The reflexion of light from the macular and peripheral fundus oculi in man. Journal of Physiology, 1952, 116, 350-356.

CROZIER, W., \& HOLWAY, A. H. Theory and measurement of visual mechanisms. I. A visual discriminationometer. II. Threshold stimulus intensity and retinal position. Journal of General Physiology, 1939, 22, 341-364.

DeGROOT, S. G., DODGE, I. M., \& SMITH, J. A. Factors in night vision sensitivity: The effect of brightness. U.S. Naval Submarine Base, New London, Report No. 194, March 18, 1952.

DeMOTT, D. W., \& BOYNTON, R. M. Retinal distribution of entoptic stray light. Journal of the Optical Society of America, 1958, 48, 13-22.

FRY, G. A. A re-evaluation of the scattering theory of glare. Illuminating Engineering, $1954,49,98-102$.

FRY, G. A., \& ALPERN, M. The effect on foveal vision produced by a spot of light on the sclera near the margin of the retina. Journal of the Optical Society of America, 1953, 43 187-188.

GLEZER, V. D. The receptive fields of the retina. Vision Research, 1965, 5, 497-525.

HAINES, R. F. Effects of high luminance sources upon the visibility of point sources. Advances in the Astronautical Sciences, 1965, 20 887-896.

HAINES, R. F. Changes in perceived size of high luminance targets. Aerospace Medicine, 1969a 40, 754-758.

HAINES, R. F. Dimensions of the apparent pupil when viewed at oblique angles. American Journal of Ophthalmology, 1969b, 68 , 649-656.

HAINES, R. F., \& ALLEN, W. H. Irradiation and manual navigation. Navigation, 1968,15 , 355-365.

HAINES, R. F., \& BARTLEY, S. H. A study of certain visual effects occasioned by factors of so-called glare. Journal of Psychology, 1966, 62, 255-266.

HAINES, R. F., \& BURGARD, J. P. Unique NASA high luminance vision laboratory uses CC techniques. Contamination Control, 1967, 6, 26-29.

HELMHOLTZ, L. F. von In J. P. C. Southall (Ed.), Helmholtz's treatise on physiological optics. Vol. 2. New York: Dover, 1962.

KATZENELLENBOGEN, E. W. Die zentrale und periphere Sehscharfe des hell- und dunkeladaptierten Auges. Psychologische Studien, 1907, 3, 272-293.

KRISTOFFERSON, A. B. Preliminary study of the sensitivity gradient of the central fovea. Project MICHIGAN, Report No. 2144-812-M (Internal Memo), June 30, 1955.

LeGRAND, Y. Recherches sur la diffusion de la lumiere dans l'oeil humain. Revue d'Optique, 1937, 16, 201-214.

LeGRAND, Y. Light, colour and vision. New York: Wiley, 1957.

LUCKIESH, M., \& MOSS, F. K. Seeing: $A$ partnership of lighting and vision. Baltimore: Williams \& Wilkins, 1931.

MANDELBAUM, J., \& SLOAN, L. L. Peripheral visual acuity. American Joumal of Ophthalmology, 1947, 30, 581-588.

MATTHEWS, M. L. Appearance of Mach bands for short durations and at sharply focused contours. Journal of the Optical Society of America, 1966, 56, 1401-1402.

McCOLLOUGH, C. The variation in width and position of Mach bands as a function of luminance.. Journal of Experimental Psychology, 1955, 49, 141-152.

OSTERBERG, G. Topography of the layer of rods and cones in the human retina. Acta Ophthalmologica (Copenhagen), Supplement 6, 1935 .

PIRENNE, M. H., \& MARRIOTT, F. H. C. Quantum theories of the absolute threshold: The influence of retinal position and area. Optica Acta, 1954, 3, 151-155.

PLATEAU, J. Memoire sur l'irradiation. Nouvelle Memoires de l'Academie de Bruxelles. Vol. 11, 1838.

POLYAK, S. The retina. (1st ed.) Chicago: University of Chicago Press, 1941.

RATLIFF, F. Mach bands: Quantitative studies on neural networks in the retina. San Francisco: Holden-Day, 1965.

RIOPELLE, A. J., \& BEVAN, W., JR. The distribution of scotopic sensitivity in human vision. American Journal of Psychology, 1953, $66,73-80$.

SCHOUTEN, J. F., \& ORNSTEIN, L. S. Measurement on direct and indirect adaptation by means of a binocular method. Journal of the Optical Society of America, 1939, 29, 168-182.

SPERLING, H. G., \& FARNSWORTH, D. Periscope acuity at night. U.S. Naval Submarine Base, New London, Report No. $157,1950$.

SPRING, K. H., \& STILES, W. S. Apparent shape and size of the pupil viewed obliquely. British Journal of Oph thalmology, 1948, 32, 347-354.

STILES, W. S. The effects of glare on the brightness difference threshold. Proceedings of the Royal Society (London), Ser. B, 1928, 104, 322-351.

VOS, J. J. On mechanisms of glare. Soesterberg, The Netherlands: Institute for Perception R VO-TNO, 1963.

WENTWORTH, H. A. A quantitative study of achromatic and chromatic sensitivity from center to periphery of the visual field. Psychological Monographs, 1930, 40(No. 3). WEYMOUTH, F. W., HINES, D. C., ACRES, L. H., RAAF, J. E., \& WHEELER, M. C. Visual acuity within the area centralis and its relation to eye movements and fixation. American Journal of Ophthalmology, 1928, 11, 947-960.

\section{NOTES}

1. For present purposes, a high contrast is one in which the target's luminance is two or more $\log _{10}$ units greater than the immediate background. 
2. Equivalent veiling luminance is measured by comparing the effect that a glare source has on the visibility or apparent brightness of a test spot, imaged at various angular separations from the glare source, with the visual effect produced by a veiling patch of light superimposed on the test spot.

3. According to Polyak's (1941) measurements, the angular distance from the center of the foveal depression in man to the rod-line, central foveal boundary, parafoveal boundary, and perifoveal boundary is $1 \mathrm{deg}$ $2 \mathrm{deg} 30 \mathrm{~min}, 3 \mathrm{deg} 20 \mathrm{~min}$, and $9 \mathrm{deg} 10 \mathrm{~min}$ of arc, respectively.

4. A basic principle related to forward scattering of light and derived from physical optics' is that if $\Phi_{0}$ is the photic flux incident upon a scattering particle, $\beta$ is the angular displacement the scattered ray makes with the direction the ray was travelling originally, and $\varphi$ is the magnitude of the photic flux scattered in direction $\beta$, then $\varphi=\Phi_{0} \cos ^{2} \beta$. For small values of $\beta$, it can be considered to be a constant and $\varphi$ becomes directly proportional to $\phi_{0}$. Therefore, the scatter curve is merely shifted by an amount proportional to the increase in target luminance. The basic reasoning remains the same for entoptic scattering. A more complete analy sis of this problem can be found elsewhere (Fry, 1954). 